\title{
Biofunctionalizing Magnetic Nanowires Toward Targeting and Killing Leukemia Cancer Cells
}

\author{
Nouf A. Alsharif ${ }^{1,2}$, Aldo I. Martínez-Banderas ${ }^{1,2}$, Jasmeen Merzaban ${ }^{1}$, Timothy Ravasi ${ }^{1}$ and Jürgen Kosel $^{2}$, \\ Member, IEEE
${ }^{1}$ King Abdullah University of Science and Technology, division of Biological and Environmental Sciences and Engineering, Thuwal, Kingdom of Saudi Arabia
${ }^{2}$ King Abdullah University of Science and Technology, division of Computer, Electrical and Mathematical Sciences and Engineering, Thuwal, Kingdom of Saudi Arabia

\begin{abstract}
The objective of targeting technologies in the field of nanomedicine is to specifically focus therapies on diseased cells in order to deliver the treatment to these cells without harming healthy ones. The use of magnetic materials in such biomedical applications offers many advantages. They can be functionalized with an agent that enhances the biocompatibility and the targeting while, at the same time, they can be controlled and monitored remotely. Iron nanowires, which usually have a native oxide shell, are specifically attractive, since they are highly biocompatible, strongly magnetic and can be coated with different biological agents. Shape anisotropy makes these nanowires permanently magnetic and therefore can be exploited for multifaceted remote manipulations, rendering them versatile nano-robots. As such, they have been used before in combination with a magnetic field to induce cancer cell death. In order to minimize the side effects of this method this study aims to enhance the targeting ability of these nanowires toward particular cells. Specifically, leukemic cells are targeted by functionalizing iron nanowires with anti-CD44 antibodies, a cell surface marker, which is overexpressed in leukemic cells compared to healthy blood cells. Iron nanowires were electrochemically fabricated with an average diameter of $35 \mathrm{~nm}$ and a length around $3 \mu \mathrm{m}$. They were coated with bovine serum albumin to facilitate their conjugation covalently with an anti-CD44 antibody by using 3-(3-dimethyl-aminopropyl) carbodiimide and of $\mathrm{N}$-hydroxysuccinimide. In order to confirm the presence of anti-CD44 antibodies on the surface of the nanowires, immunostaining, and Fourier transform infrared spectroscopy were used. In addition, cytotoxicity effects of bare iron nanowires, coated and functionalized nanowires were studied by using cell proliferation assays. These studies illustrated that the nanowires have a high level of biocompatibility.
\end{abstract}

Index Terms-Antibodies, Functionalization, Iron, Nanowires.

\section{INTRODUCTION}

The use of magnetic nanostructures for biomedical applications has significantly increased in the last few years. Magnetic nanowires (NWs) have similar advantages as commonly used superparamagnetic iron oxide nanoparticles [1], when it comes to biocompatibility [2] and functionalization [3]. Their length and diameter can be easily modified, depending on the method of fabrication and the materials available [4]. The shape of the NWs has two important consequences, changing the properties compared to the nanoparticles: 1) the NWs have larger remnant magnetic moments, which enhance the remote controllability of their movement by magnetic fields [5], [6]. This property has been used to induce cancer cell death by a magneto-mechanical effect. 2) The larger surface area to volume ratios, which increases the capabilities for surface modifications [7]-[10]. In this context, modifying the NWs' surface can provide attachment links for functional agents, such as antibodies, which enhance the specific targeting [11], drugs [10], or dyes [12].

Although a large number of studies have focused on Nickel NWs, these NWs have been shown to have genotoxic and cytotoxic effects [13], [14]. In contrast, iron NWs (Fe NWs) have shown a high level of biocompatibility, even at high concentrations and long incubation times with the cells [9], [10], [15]. The iron oxide shell, that forms either as a native passivation or by annealing [2], plays a major role in this biocompatibility [10]. While it can also affect the NW's magnetization [2], this effect usually plays a minor role, due to the small amount of volume oxidized.

In a study of Fe NWs loaded with the chemotherapeutic drug, doxorubicin, it was shown that cancer cells were killed efficiently by drug delivery in combination with magnetomechanical stimulation [10]. In order to provide a selective treatment and minimize side effects to healthy cells, functionalization of the Fe NWs, e.g. with antibodies, to target cell surface receptors, is required.

Acute myeloid leukemia is a blood cancer caused by genetic defects in hematopoietic progenitor cells (HPCs). These genetic changes block the differentiation pathway of HPCs, resulting in immature non-functional blood cells that possess an uncontrolled proliferation [16]. In addition, CD44 cell surface markers are highly expressed on leukemic cells, compared to normal blood cells [17]. Thus, the goal of this study was to functionalize Fe NWs with monoclonal antiCD44 antibodies to target CD44 markers on leukemic cells. This approach is expected to significantly increase the probability of specific targeting of cancer cells without harming healthy cells. 


\section{EXPERIMENTAL METHODS}

\section{A. Nanowire fabrication}

The fabrication of Fe NWs is based on electrodeposition into alumina templates, which is accomplished in six steps. First, a pure aluminum disc (GoodFellow), with a diameter of $2.5 \mathrm{~mm}$ and a thickness of $0.5 \mathrm{~mm}$ was electropolished by using a $70 \%$ perchloric acid solution with a voltage of $20 \mathrm{~V}$. Then, an anodization was carried out with $0.3 \mathrm{M}$ oxalic acid at $4^{\circ} \mathrm{C}$, applying a voltage of $40 \mathrm{~V}$ for $24 \mathrm{~h}$. This step grows an alumina layer and establishes pores that unorganized in arrangement and diameter at the surface, but regularly aligned at the bottom. After that, the membrane was incubated in 0.2 $\mathrm{M}$ chromium trioxide $\left(\mathrm{CrO}_{3}\right)$ for $12 \mathrm{~h}$ at $40{ }^{\circ} \mathrm{C}$, in order to remove the alumina layer. This leaves aluminum disc with hexagonally ordered indentations. In the fourth step, asecond anodization grows another alumina layer with uniformly arranged pores that originated from the indentations. This anodization step is carried out for $4 \mathrm{~h}$ using the same conditions as the first anodization and resulting in $5 \mu \mathrm{m}$ deep pores. Thereafter, the bottoms of the pores were connected to the aluminum substrate via the growth of dendrites. before the last step, the template was filled with iron solution, that made of $13.9 \mathrm{~g}$ Iron sulfate $\left(\mathrm{FeSO}_{4}\right), 7.45 \mathrm{~g}$ sodium sulfate $\left(\mathrm{Na}_{2} \mathrm{SO}_{4}\right), 2.47 \mathrm{~g}$ Boric acid $\left(\mathrm{H}_{3} \mathrm{BO}_{3}\right)$ and $0.16 \mathrm{~g}$ Ascorbic acid $\left(\mathrm{C}_{6} \mathrm{H}_{8} \mathrm{O}_{6}\right)$ dissolved in $100 \mathrm{ml}$ distilled water. Finally, the NWs were grown by applying pulsed electrodeposition (-60 mA pulses of $2 \mathrm{~ms}$ duration) for $1 \mathrm{~h}$ at room temperature. The full explanation of the fabrication process can be found in previous studies [9], [10], [12], [18], [19].

In order to release the NWs from the alumina, it was cut into pieces that were inserted into an Eppendorf tube and immersed in $1 \mathrm{ml}$ of $1 \mathrm{M}$ sodium hydroxide for $1 \mathrm{~h}$. Then, the $\mathrm{Al}$ template pieces were removed and the solution was renewed every hour for four times with $10 \mathrm{~s}$ of sonication in-between. To clean the NWs from the sodium hydroxide, they were rinsed six times using $96 \%$ ethanol with $10 \mathrm{~s}$ of sonication and then stored in the solution for the experiments [9], [10], [12]. The number of NWs contained in one disc was obtained by mixing $10 \mu \mathrm{l}$ of the solution with the released NWs with 300 $\mu \mathrm{l}$ Hydrochloric acid (37\%) and incubating it for $20 \mathrm{~min}$. Then, the volume was completed to $3 \mathrm{ml}$ by adding distilled water. Inductively coupled plasma mass spectrometry was then used to quantify the Fe NW concentration. The schematic in (FIG.1A) present a summary of the NW fabrication process.

The morphology of Fe NWs was characterized by scanning electron microscopy (SEM, Quanta 3D; FEI Company, Hillsboro, OR, USA) and transmission electron microscopy (TEM, Tecnai BioTWIN; FEI Company) was used to determine the diameter, length and surface coating of the NWs. Electron energy loss spectroscopy (EELS) was used to characterized the iron (core) and iron oxide (shell). The samples for SEM, TEM, and EELS were prepared by taking 2 $\mu \mathrm{l}$ of NW solution in ethanol and placing it on a silicon substrate for SEM and on a copper-carbon mesh substrate for TEM. Then, the samples were investigated after complete drying. Moreover, the magnetic properties of the Fe NWs

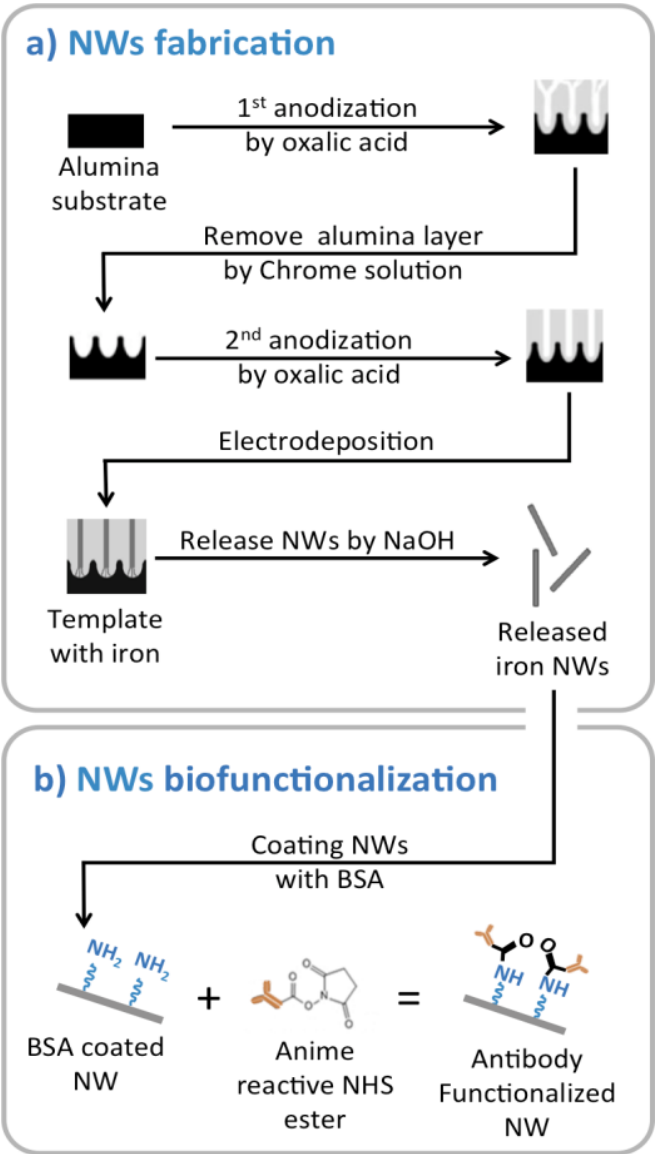

Fig. 1. Schematics of a) the fabrication process of iron nanowires and b) coating of the nanowires with BSA followed by biofunctionalization with antibodies.

were characterized by measuring their hysteresis curves with a vibrating sample magnetometer (VSM).

\section{B. Nanowires coating and functionalization}

Fe NWs were coated with bovine serum albumen (BSA) by washing released Fe NWs three times with $10 \mathrm{mM}$ phosphate buffer (PB) of $\mathrm{pH}$ 7.4. Then, they were immersed into BSA with a concentration (22 mg BSA/1 mg NWs) for $1.5 \mathrm{~h}$ under sonication. After that, the NWs were washed four times with (PB) and stored at $4{ }^{\circ} \mathrm{C}$ for the next use.

The NW functionalization is based on a covalent conjugation between the amine group on the BSA coated NWs (i.e., BSA NWs) and the NHS ester group on the activated antibody. 990 $\mu \mathrm{l}$ of $25 \mu \mathrm{g} / \mathrm{mL}$ anti-CD44 antibody clone Hermes-3 (ABGENT) were incubated with $10 \mu \mathrm{l}$ of fresh and cold crosslinking solutions for $20 \mathrm{~min}$. This solution was prepared by dissolving $8 \mathrm{mg}$ of 3-(3-dimethyl-aminopropyl) carbodiimide (EDC) and $22 \mathrm{mg}$ of $\mathrm{N}$-hydroxysuccinimide (NHS) in $1 \mathrm{~mL}$ of 2-(N-Morpholino) ethanesulfonic acid hydrate (MES; 0.1 M pH 4.7). This interaction activates the antibody carboxyl groups. Then, the washed BSA NWs were incubated with activated antibodies for $2 \mathrm{~h}$ at room temperature. Finally, the functionalized Fe NWs (i.e., AbNWs) were rinsed, blocked with $2 \% \mathrm{BSA}$ and stored at $4{ }^{\circ} \mathrm{C}$. The biofunctionalization process is summarized in (FIG.1B). 


\section{Immunostaining}

To confirm the antibody immobilization at the surface of the NWs, both BSA-NWs and Ab-NWs were incubated with 50 $\mathrm{mg} / \mathrm{ml}$ of green fluorescent-labeled secondary antibody (DyLight 488 Goat anti-Mouse IgG $(\mathrm{H}+\mathrm{L})$ : Thermo Fisher) for $1 \mathrm{~h}$ under $300 \mathrm{rpm}$ shaking. The NWs were rinsed three times with $\mathrm{PB}$, placed on a glass microscope slide and sandwiched from the top by a coverslip. The images were investigated under the laser scanning confocal microscope (MTZ02 - Leica TCS SP5) using 63x oil lens and ALEXA 488 laser.

\section{Fourier Transform Infrared Spectroscopy (FTIR)}

In order to analyze the characteristics of the functionalized Fe NWs, a similar amount of Fe-NWs, BSA-NWs, and AbNWs were added on gold-coated microscope slides (Thermo Fisher Scientific) and left to dry for $5 \mathrm{~min}$. at room temperature. The samples were detected with a Nicolet Continuum FT-IR microscope and OMIC software was used to analyze the data.

\section{E. Cell culturing}

An acute myeloid leukemia cell line HL60 (ATCC® CCL $\neg 240^{\mathrm{TM}}$ ) was cultured in RPMI 1640 with $10 \%$ fetal bovine serum (FBS) and $5 \mathrm{ml}$ penicillin-streptomycin. All of the supplements and the media were bought from (GIBCO ${ }^{\circledR}$ life technologies). The cells were grown inside the incubator under a humidified environment with $5 \% \mathrm{CO} 2$ at $37{ }^{\circ} \mathrm{C}$.

\section{F. Biocompatibility study}

Leukemic cells were grown in 96-flat well plates with a concentration of $100000 \mathrm{cell} / \mathrm{ml}$. Before treating the cells, the NWs were washed three times with PB followed by four washing steps with warmed RPMI media. $250 \mathrm{NWs}$ /cell (i.e., $7.42 \mathrm{E}-04 \mu \mathrm{g} \mathrm{Fe} / \mathrm{ml}$ ) were added per well and incubated with the cells for $24 \mathrm{~h}$. The XTT cell proliferation assay was applied to the cells by following the kit protocol (TACS $®$ XTT Cell Proliferation Assay Cat\# 4891-025-K, 2500 Tests). After warming up both XTT reagent and the activator at 37 ${ }^{\circ} \mathrm{C}, 100 \mu \mathrm{l}$ of the XTT activator were mixed with $5 \mathrm{~mL}$ of XTT reagent. Then $50 \mu \mathrm{l}$ of the prepared XTT working solution were added to each well and incubated for $2 \mathrm{~h}$ at 37 ${ }^{\circ} \mathrm{C}$. The result was obtained by reading the 96 -well plate in the microplate reader at $490 \mathrm{~nm}$ with a reference wavelength of $630 \mathrm{~nm}$. All the experiments have been done in triplicates.

\section{RESULTS AND DISCUSSION}

\section{A. Nanowires characterization}

Inductively coupled plasma mass spectrometry results revealed that each alumina disc contained around $0.6 \mu \mathrm{g}$ of NWs, which corresponds to $5.6 \mathrm{E}+10 \mathrm{NWs}$ of $40 \mathrm{~nm}$ in diameter and $3 \mu \mathrm{m}$ in length. The morphology of the released NWs is shown in FIG. 2A and FIG. 3A. In FIG. 3A an iron oxide layer surrounding the NWs can be seen, which has previously been identified as $\mathrm{Fe}_{2} \mathrm{O}_{3}$ phase [10]. The thickness of this layer is around $3.5 \mathrm{~nm}$, which is formed during and after the release step [8], due to the exposure to the air, while releasing and washing with absolute ethanol [5]. This oxide interphase can be used for the attachment of coating agents to the NWs' surface.

VSM has been used to characterize the magnetic properties of Fe NWs embedded in the alumina template. FIG. 2B shows the hysteresis loop, with a saturation magnetization $\left(\mathrm{M}_{\mathrm{s}}\right)$ of $427 \mathrm{~A} . \mathrm{m}^{2} / \mathrm{kg}$ and a coercive field of $\left(\mathrm{H}_{\mathrm{c}}\right) 138.4 \mathrm{kA} / \mathrm{m}$ for the field applied parallel to the NWs (easy axis). The large remanent magnetization $\left(\mathrm{M}_{\mathrm{R}}\right)$ of $388 \mathrm{~A} \cdot \mathrm{m}^{2} / \mathrm{kg}$, confirms the permanent magnetic properties of the NWs.
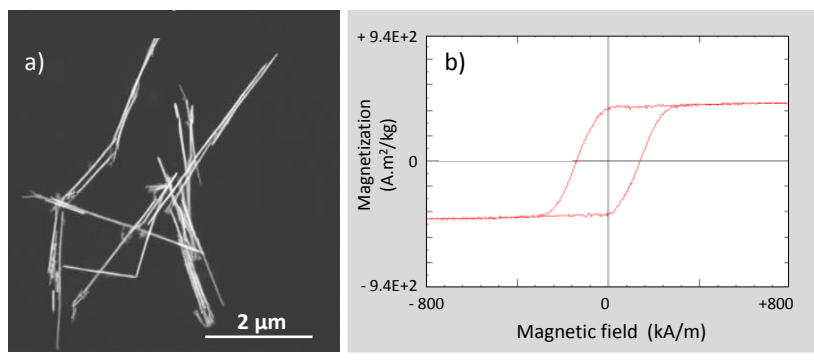

Fig. 2. a) SEM image of iron nanowires. b) Magnetization loop (VSM) of an array of iron nanowires embedded in the alumina template.

\section{B. Nanowire coating and functionalization}

In order to immobilize the biological agents, i.e., antibodies, on the Fe NWs, amine groups on the surface of the NWs are utilized. This has been achieved by coating the NWs with BSA. The coating was confirmed by TEM imaging (FIG. 3B). To determine the immobilization of anti-CD44 antibodies onto the Fe NWs, two approaches were applied. The first one was using secondary antibodies that target only functionalized NWs. A strong signal observed on Ab-NWs (FIG. 3C) and no signal from non-functionalized, i.e. BSA NWs, (FIG. 3D) confirms the functionalization.
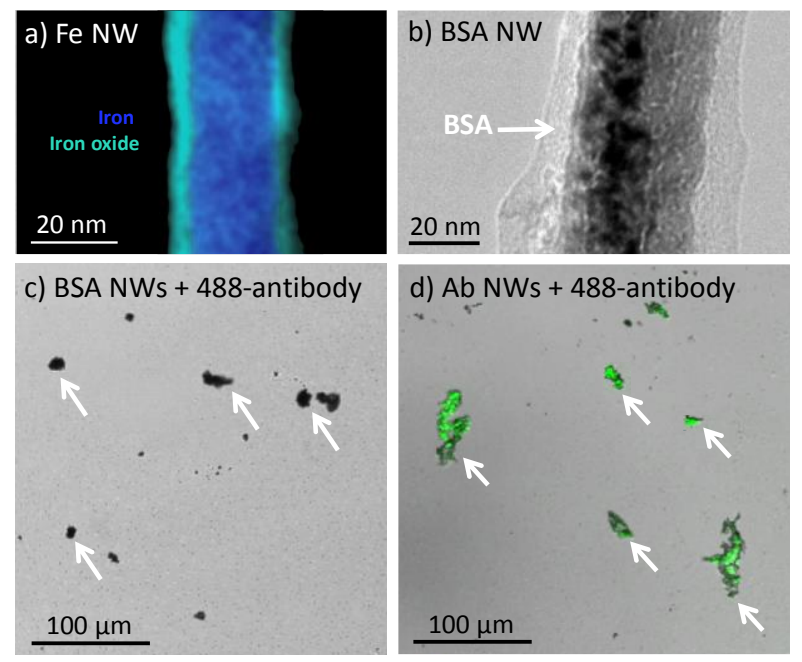

Fig. 3. a) EELS image of iron nanowire before BSA coating that shows the average thickness of the iron oxide layer $=3.5 \mathrm{~nm}$. b) TEM image of iron nanowire after BSA coating. c) Clusters of BSA-NWs conjugated to labeled secondary antibodies (ALEX 488) and d) clusters of Ab-NWs conjugated to labeled secondary antibodies (ALEX 488). 


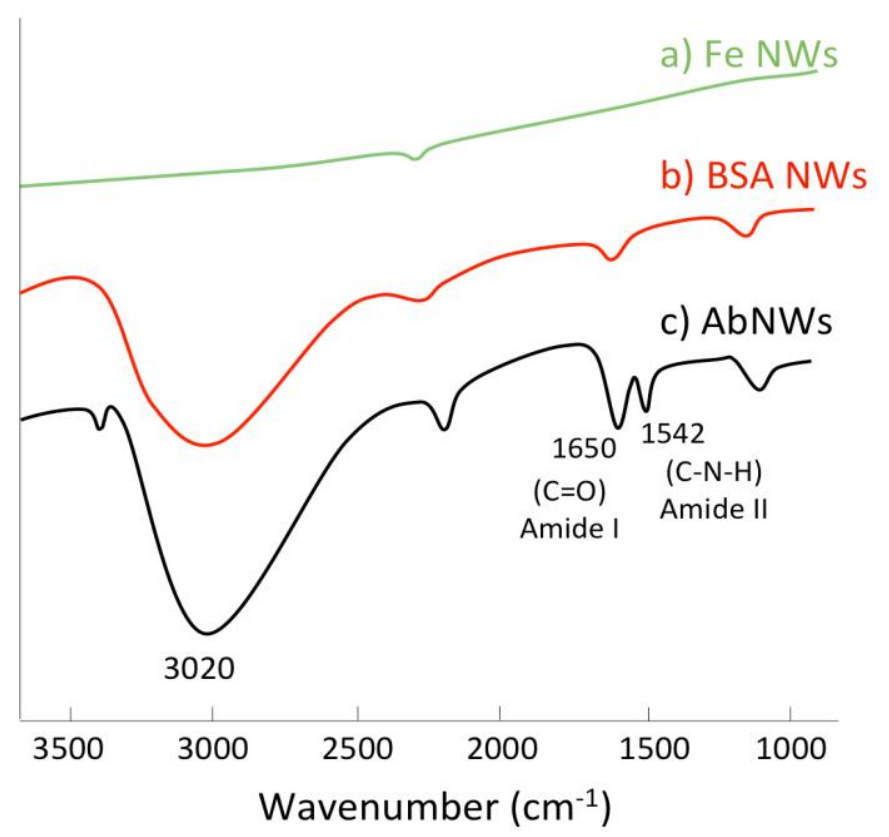

Fig. 4. Fourier transform infrared spectra of coated and functionalized NWs in the $1000-3500 \mathrm{~cm}^{-1}$ region. a) Iron NWs, b) BSA coated NWs, which present a spectra with absorption at $3018 \mathrm{~cm}^{-1}$ and $1651 \mathrm{~cm}^{-1}$ for hydroxide $(\mathrm{O}-\mathrm{H})$ and amide I $(\mathrm{C}=\mathrm{O})$ groups, respectively. c) biofunctionalized $\mathrm{NWs}$ show absorptions in the spectra at $3020 \mathrm{~cm}^{-1}, 1650 \mathrm{~cm}^{-1}$, and $1542 \mathrm{~cm}^{-1}$ for $(\mathrm{O}-\mathrm{H})$ hydroxide, $(\mathrm{C}=\mathrm{O})$ amide $\mathrm{I}$ and $(\mathrm{C}-\mathrm{N}-\mathrm{H})$ amide II groups, respectively.

The second method was using Fourier Transform Infrared Spectroscopy in order to detect the functional groups that were formed after the antibody attachment. In theory, an amide group (R-CO-NH) should be created after the interaction between carboxyl and ester groups [20]. Indeed, as shown in FIG.4C, the formation of the amide group is obtained in the Ab-NWs. The signal at $1542 \mathrm{~cm}^{-1}$ stems from amide II (C-N$\mathrm{H})$ groups [21] and is only found in the case of Ab-NWs. However, the peak at $1650 \mathrm{~cm}^{-1}$ was observed in both BSA-

NWs and Ab-NWs and is attributed to the presence of amide I $(\mathrm{C}=\mathrm{O})$ groups [21], [22]. The peaks at $3018 \mathrm{~cm}^{-1}$ and 3020 $\mathrm{cm}^{-1}$ in both BSA-NWs and Ab-NWs, respectively, are attributed to the stretching of Hydroxide $(\mathrm{O}-\mathrm{H})$ groups [21], [22] that come from the BSA. These results confirm the immobilization of antibodies on the NWs.

\section{Nanowire Biocompatibility}

The cell viability assay, XTT, is based on the reduction of the tetrazolium salt 2,3-Bis (2-methoxy-4-nitro-5-sulfophenyl)2H-tetrazolium-5-carbanilide) to formazan which occurs through the succinate-tetrazolium reductase system in the mitochondria of metabolically active cells. The reaction is attributed mainly to mitochondrial enzymes and electron carriers [23].

In our case the XTT assay was used to study the toxicity of the NWs on leukemic cells. As shown in FIG. 5, both BSANWs and Ab-NWs have a high level of biocompatibility compared to Fe-NWs. However, there is no significant decrease between Fe-NWs and cells without any treatment, but it seems that the BSA and antibody coating further reduces the toxicity of the Fe NWs., which is already very low.

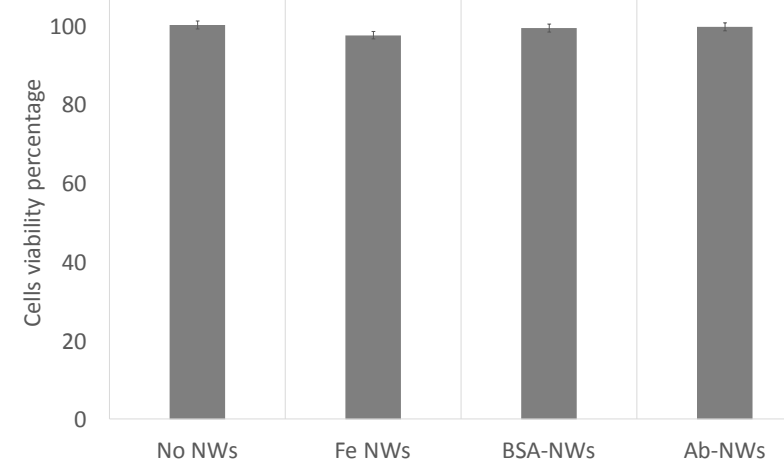

Fig. 5. Cell viability assay (XTT) after treatment of leukemic cells with iron NWs (Fe-NWs), BSA coated NWs (BSA-NWs) and anti-CD44 antibody coated NWs (Ab-NWs) for 24h. (No NWs) is the positive control, where cells are growing without NWs and the cell viability value is $100 \%$. The data represent means \pm standard deviation, $n$ $=3$.

\section{CONCLUSION}

Iron NWs have been fabricated by electrochemical deposition into alumina templates. A method for coating them with BSA and functionalization with anti-CD44 antibodies was developed. BSA was used as a linker molecule facilitating the attachment of anti-CD44 antibodies to the iron oxide shell of the NWs, which was confirmed by TEM image that showed a continuous layer of BSA around the NWs. In addition, Immunostaining and FTIR confirmed the successful functionalization of the NWs with the antibodies. Combining these NWs with leukemic cancer cells showed a high level of biocompatibility of both BSA-NWs and Ab-NWs in vitro. Overall, the high biocompatibility of functionalized NWs and the ability of remotely controlling their movement by using the magnetic field, make them a promising platform for targeted treatments.

\section{ACKNOWLEDGMENT}

Research reported in this publication was supported by the King Abdullah University of Science and Technology (KAUST).

\section{REFERENCES}

K. M. Krishnan, "Biomedical nanomagnetics: a spin through possibilities in imaging, diagnostics, and therapy," IEEE transactions on magnetics, vol. 46, no. 7, pp. 2523-2558, 2010.

[2] Y. P. Ivanov, A. Alfadhel, M. Alnassar, J. E. Perez, M. Vazquez, A. Chuvilin, and J. Kosel, "Tunable magnetic nanowires for biomedical and harsh environment applications," Scientific reports, vol. 6, pp. 24189, 2016.

[3] A. Sharma, G. M. Orlowski, Y. Zhu, D. Shore, S. Y. Kim, M. D. DiVito, A. Hubel, and B. J. H. Stadler, "Inducing cells to disperse nickel nanowires via integrin-mediated responses," Nanotechnology, vol. 26, no. 13, pp. 135102, 2015. 
[4] V. K. Varadan, L. Chen, and J. Xie, Nanomedicine: design and applications of magnetic nanomaterials, nanosensors and nanosystems, 1st ed., vol 1., John Wiley \& Sons, 2008, pp.3-13

[5] A. Hultgren, M. Tanase, C. S. Chen, G. J. Meyer, and D. H. Reich, "Cell manipulation using magnetic nanowires," Journal of Applied Physics, vol. 93, no. 10, pp. 7554-7556, 2003.

[6] A. Hultgren, M. Tanase, E. J. Felton, K. Bhadriraju, A. K. Salem, C. S. Chen, and D. H. Reich, "Optimization of yield in magnetic cell separations using nickel nanowires of different lengths," Biotechnology progress, vol. 21, no. 2, pp. 509-515, 2005.

[7] S. S. Banerjee, and D.-H. Chen, "Multifunctional pH-sensitive magnetic nanoparticles for simultaneous imaging, sensing and targeted intracellular anticancer drug delivery," Nanotechnology, vol. 19, no. 50, pp. 505104, 2008.

[8] G. Bhabra, A. Sood, B. Fisher, L. Cartwright, M. Saunders, W. H Evans, A. Surprenant, G. Lopez-Castejon, S. Mann, and S. A. Davis, "Nanoparticles can cause DNA damage across a cellular barrier," Nature nanotechnology, vol. 4, no. 12, pp. 876, 2009.

[9] M. F. Contreras, "Magnetic Nanowires as Materials for Cancer Cell Destruction," Ph.D. dissertation, Biological scince, King Abdullah University of Science and Technology, Saudi arabia , 2015, puplished.

[10] A. I. Martínez-Banderas, A. Aires, F. J. Teran, J. E. Perez, J. F. Cadenas, N. Alsharif, T. Ravasi, A. L. Cortajarena, and J. Kosel, "Functionalized magnetic nanowires for chemical and magnetomechanical induction of cancer cell death," Scientific reports, vol. 6, pp. 35786, 2016.

[11] M. F. Contreras, T. Ravasi, and J. Kosel, "Targeted cancer cell death induced by biofunctionalized magnetic nanowires.", IEEE, Middle East Conference in Biomedical Engineering (MECBME), (pp. 47-50), 2014.

[12] M. B. Margineanu, K. Julfakyan, C. Sommer, J. E. Perez, M. F. Contreras, N. Khashab, J. Kosel, and T. Ravasi, "Semi-automated quantification of living cells with internalized nanostructures," Journal of nanobiotechnology, vol. 14, no. 1, pp. 4, 2016.
M. F. Contreras, A. Zaher, J. E. Perez, T. Ravasi, and J. Kosel, "Magnetic nanowires and hyperthermia: How geometry and material affect heat production efficiency.", IEEE, InMagnetics Conference (INTERMAG), (pp. 1-1), May 2015

[14] K. S. Kasprzak, F. W. Sunderman, and K. Salnikow, "Nickel carcinogenesis," Mutation Research/Fundamental and Molecular Mechanisms of Mutagenesis, vol. 533, no. 1, pp. 67-97, 2003.

[15] C.-B. Wang, and W.-X. Zhang, "Synthesizing nanoscale iron particles for rapid and complete dechlorination of TCE and PCBs," Environmental science \& technology, vol. 31, no. 7, pp. 21542156, 1997.

[16] R.-S. Charrad, Y. Li, B. Delpech, N. Balitrand, D. Clay, C. Jasmin, C. Chomienne, and F. Smadja-Joffe, "Ligation of the CD44 adhesion molecule reverses blockage of differentiation in human acute myeloid leukemia," Nature medicine, vol. 5, no. 6, pp. 669676, 1999.

[17] L. Jin, K. J. Hope, Q. Zhai, F. Smadja-Joffe, and J. E. Dick, "Targeting of CD44 eradicates human acute myeloid leukemic stem cells," Nature medicine, vol. 12, no. 10, pp. 1167, 2006.

[18] H. Masuda, and K. Fukuda, "Ordered metal nanohole arrays made by a two-step replication of honeycomb structures of anodic alumina," science, vol. 268, no. 5216, pp. 1466-1468, 1995.

[19] M. F. Contreras, A. Zaher, J. E. Perez, T. Ravasi, and J. Kosel, "Magnetic nanowires and hyperthermia: How geometry and material affect heat production efficiency." pp. 1-1.

[20] C. L. Allen, and J. M. J. Williams, "Metal-catalysed approaches to amide bond formation," Chemical Society Reviews, vol. 40, no. 7, pp. 3405-3415, 2011.

[21] X. Mu, C. Yan, Q. Tian, J. Lin, and S. Yang, "Bsa-assisted synthesis of ultrasmall gallic acid-Fe (III) coordination polymer nanoparticles for cancer theranostics," International journal of nanomedicine, vol. 12, pp. 7207, 2017.

[22] D. K. Bora, and P. Deb, "Fatty acid binding domain mediated conjugation of ultrafine magnetic nanoparticles with albumin protein," Nanoscale research letters, vol. 4, no. 2, pp. 138, 2009.

[23] ATCC, "XTT and MTT cell prolifreation assay kits," ATCC maual, p. 2. , 2018. 